\title{
An analysis of myotonia in paramyotonia congenita ${ }^{1}$
}

\author{
DAVID BURKE, NEVELL F. SKUSE, AND A. KEITH LETHLEAN \\ From the Unit of Clinical Neurophysiology, Division of Neurology, \\ Prince Henry Hospital, Little Bay, N.S.W. 2036, Australia
}

SYNOPSIS In two subjects with paramyotonia congenita myotonic delay in muscle relaxation, recorded electromyographically and with a displacement transducer, was found to increase with repeated forceful contractions. Myotonia was elicited readily in warm temperatures, was initially aggravated by cooling, but was invariably lost as muscle fatigue developed. The EMG evidence of myotonia usually subsided before complete muscle relaxation had occurred, suggesting that a defect of the contractile mechanism was present over and above any defect at membrane level.

The non-dystrophic forms of myotonia may be distinguished one from the other on the basis of heredity and the patterns of myotonia and of weakness. Paramyotonia congenita is said to be characterized by 'paradoxical' myotonia which is accentuated by repetitive muscle contraction, and by extreme sensitivity to cooling, which aggravates the myotonia and the muscle weakness. But paradoxical myotonia has not been a uniform finding in all patients with otherwise classical paramyotonia, and it has even been reported to be present in some but absent in other members of the same family. While these discrepancies may arise in part from differing degrees of severity in different patients examined under different circumstances, this explanation is unsatisfactory if the paradoxical nature of the myotonia is to be used as a feature distinguishing paramyotonia congenita from the dominant and recessive forms of myotonia congenita.

This paper analyses aspects of myotonia in two subjects with paramyotonia congenita with particular reference to the responses to repeated muscle contraction and to muscle cooling. Muscular weakness has been analysed in a companion paper (Burke et al., 1974b).

\section{METHODS}

Data were obtained from 13 experimental sessions in a 25 year old subject with classical paramyotonia congenita and were confirmed in a further experi1 Some of these findings were reported to the 1974 meeting of the Australian Association of Neurologists. mental session in a similarly afflicted 19 year old brother. These sessions provided data for this paper and a companion paper on muscular weakness (Burke et al., 1974b). Clinically the myotonia in both subjects was 'paradoxical'.

Most experiments were performed on the abductor digiti minimi muscle (ADM) but one experimental session was devoted to the triceps surae, from which similar data were obtained. In these two muscles, voluntary and electrically-induced contractions were studied under isometric and isotonic conditions. In four experiments voluntary contractions of the flexor digitorum profundus (FDP) were studied under isotonic conditions. Isometric contractions of ADM and of triceps surae were elicited as described by Burke et al. $(1974 \mathrm{a}, \mathrm{b})$. The displacement produced by isotonic contractions was recorded by a Burdick FM.1 Photomotograph, positioned so that the resulting movement interrupted the light beam from a photoelectric cell. For ADM the hand was fixed in a frame with a horizontal bar which prevented finger flexion. The fifth finger fitted into a retaining ring which could move freely in the horizontal plane so that abduction movements were not impeded. A lightweight spring of low tensile strength was attached to the retaining ring to return the abducted finger to the control position once contraction had subsided. For triceps surae the patient lay prone so that the plantar flexion movement was performed against gravity which returned the foot to the control position as the contraction subsided. For FDP finger flexion was opposed by a light-weight spring which was adjusted so that it was capable of extending the fingers when they were completely relaxed.

The electromyogram (EMG) was recorded by surface electrodes taped to the bellies of the muscles. 
For FDP and for some experiments on ADM intramuscular EMG was recorded using a concentric needle electrode (Disa 9013KO512). Experiments were carried out in a warm air-conditioned laboratory. The skin temperature over ADM, triceps surae, and FDP was measured by an Ellab electronic thermometer and was maintained at $34-35^{\circ} \mathrm{C}$. The hand and arm were cooled by packing a plastic bag containing ice around the limb.

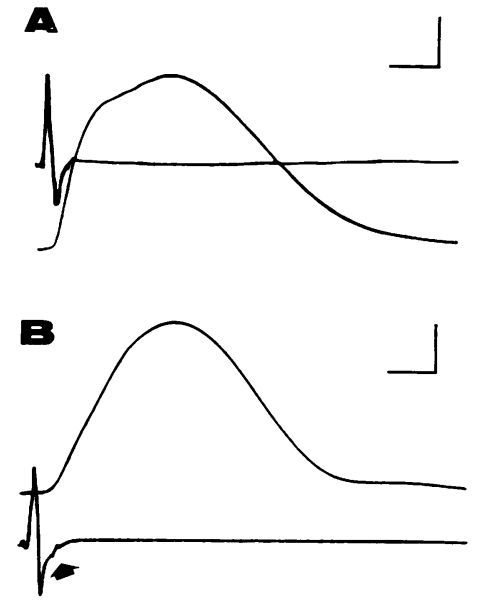

FIG. 1. Twitch contractions of triceps surae. A: isometric contraction of right triceps surae. B: isotonic contraction of left triceps surae. Note the small $H$ wave, indicated in B by the arrow. Calibrations: horizontal-50 $\mathrm{ms}$ for $\mathrm{A}$ and $\mathrm{B}$; vertical-9.32 $\times 10^{-3}$ $\mathrm{Nm}$ for A, $6 \mathrm{mV}$ for $\mathrm{A}$ and $\mathrm{B}$.

\section{RESULTS}

Clinically, myotonia in the form of a persistent failure of muscle relaxation could be readily elicited at surface temperatures of $34-35^{\circ} \mathrm{C}$. In unfatigued muscle this myotonia became more prominent with repeated voluntary contraction of muscle. Voluntary isotonic contraction appeared to induce myotonia more readily than voluntary isometric contraction in which the contracting muscle was prevented from shortening. After vigorous isometric contraction, removal of the restraint often resulted in an abduction movement of the fifth finger (with ADM) or flexion of the fingers (with FDP), thus shortening the appropriate muscle. However clinically it appeared that the force then necessary to over- come this involuntary stiffness was less than that required if the muscle had been allowed to shorten in an isotonic contraction.

ADM, FDP, and abductor pollicis brevis were sampled with concentric needle electrodes. Increased insertional activity was recorded, with spontaneous activity of fibrillation or positive sharp wave form. Recurring volleys of sharp waves were seen to occur apparently spon-

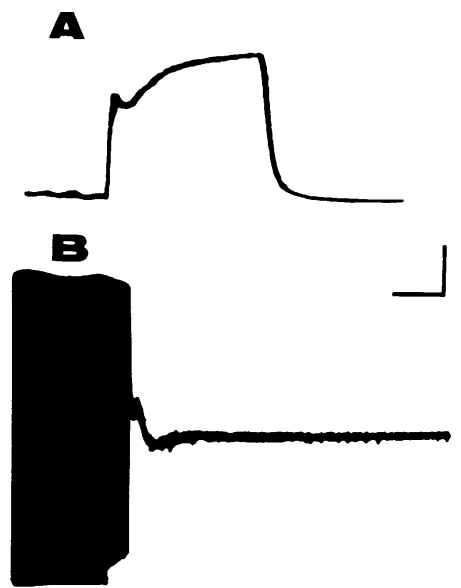

FIG. 2. Isotonic contraction of $A D M$ induced by tetanic stimulation at $50 \mathrm{~Hz}$. A: time course of contraction. There is no delay in relaxation on cessation of stimulation. B: EMG during and after stimulation. The EMG sweep was started after tetanization had been in progress for $1 \mathrm{~s}$. No myotonic activity is seen. Calibrations: horizontal-1 s for A and $\mathrm{B}$; vertical$150 \mu \mathrm{V}$ for B.

taneously or to be evoked by electrode movement or voluntary contraction. Motor unit action potentials were within normal limits but the interference pattern fatigued rapidly on sustained effort. Cessation of voluntary contraction was followed by continued electrical activity in the form of motor unit potentials, fibrillation potentials and positive sharp waves.

ADEQUATE STIMULUS FOR MYOTONIA Electricallyinduced contractions failed to provoke myotonia in ADM and in triceps surae. In ADM, isometric and isotonic twitch contractions had similar time courses-contraction time 63-70 $\mathrm{ms}$, and half-relaxation time $68-80 \mathrm{~ms}$ (c.f. Fig. 
A
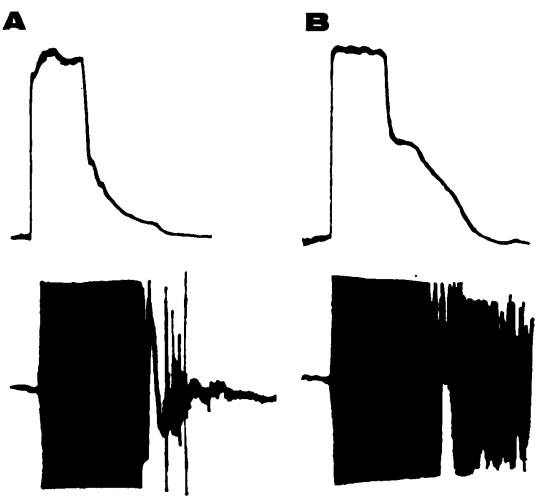
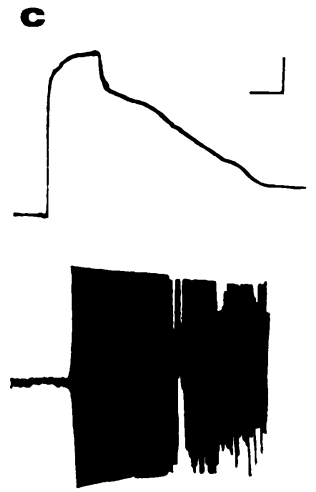

FIG. 3. Isotonic voluntary contraction of $A D M . \mathrm{A}, \mathrm{B}$, and $\mathrm{C}$ represent the first, third, and fifth contractions of a series of maximal isotonic voluntary contractions. There is increasing slowness of relaxation (myotonia) in successive contractions as seen in the displacement transducer records (upper traces) and the accompanying EMG records (lower traces). Note that the time bases differ for the displacement and EMG records. Calibrations: horizontal-2 s for displacement traces, $1 \mathrm{~s}$ for EMG traces; vertical$150 \mu V$ for EMG traces.

4, Burke et al., 1974b). Isometric twitch contractions of triceps surae (Fig. 1A) had a slurred rising phase at approximately $50-60 \mathrm{~ms}$ (presumably due to the fast twitch gastrocnemii) with a peak at $120 \mathrm{~ms}$ (presumably due to the slow twitch soleus) and a half-relaxation time of $105 \mathrm{~ms}$. Isotonic contractions of triceps surae were of smooth contour (Fig. 1B), having a contraction time of $135 \mathrm{~ms}$ and a half-relaxation time of $98 \mathrm{~ms}$, which values fall within normal limits for this method (Preswick et al., 1966). In both muscles the relaxation phase after each contraction followed a normally smooth course, and no repetitive electrical activity was seen in the surface EMG at amplifications of $150 \mu \mathrm{V} / \mathrm{cm}$. Trains of repetitive stimuli at different frequencies similarly failed to induce evidence of myotonia.

Supramaximal stimulation at $50 \mathrm{~Hz}$ was performed using brief trains of five impulses, and prolonged trains of duration 1, 2, 5, 10, and $30 \mathrm{~s}$. Myotonia was not visible in the surface EMG at amplifications of $150 \mu \mathrm{V} / \mathrm{cm}$ or even $60 \mu \mathrm{V} / \mathrm{cm}$, whether the preceding contraction had been isotonic or isometric (Fig. 2B), so that relaxation on cessation of muscle contraction appeared to be rapid and smooth (Fig. 2A, see also Figs 2 and 3 in Burke et al., 1974b). Repeated trains of 2-5 s duration similarly did not induce visible evidence of myotonia.

Forceful voluntary contraction induced repetitive EMG activity on relaxation, with a concomitant delay in muscle relaxation (Fig. 3). This evidence of myotonia could be elicited almost invariably with isotonic contractions of the unfatigued ADM, but was often not found in the initial contractions of a series of isometric contractions (c.f. Fig. 1, Burke et al., 1974b). If voluntary contraction was alternated with tetanic contraction, myotonia could be elicitedo with the former but not the latter.

In two experiments intravenous injection of edrophonium $10 \mathrm{mg}$ did not produce spon@ taneous myotonia in relaxed muscles, althougle mild muscle contraction more readily induced myotonia than before the injection. Blinking then produced blepharospasm which could be controlled only by the subject's conscious efforts $\vec{A}$ to relax.

NATURE OF MYOTONIA In an unfatigued muscle the clinical, mechanical, and electrical evidence of myotonia increased with repeated voluntary contractions under both isotonic and isometric conditions (Figs 3,4). With the onset of muscular fatigue, manifested in ADM by decreased amplitude of the interference pattern and a lower maximum torque or a smaller and less well maintained excursion of the fifth finger, the myotonia no longer appeared to be 'paradoxical'. In the fatigued state it was common to have no evidence of myotonia.

In ADM, particularly with isotonic contractions, it was often noted that the mechanical evidence of myotonia outlasted the electrical evidence as recorded by surface electrodes at amplifications of $150 \mu \mathrm{V} / \mathrm{cm}$ or $60 \mu \mathrm{V} / \mathrm{cm}$. By the time the visible EMG activity had subsided completely the fifth finger had not yet returned to its control position and appeared clinically to 


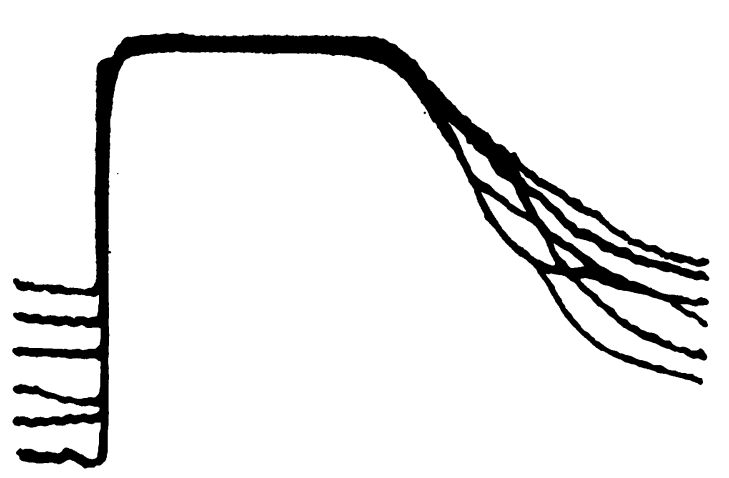

FIG. 4. Isotonic voluntary contraction of ADM. Six successive isotonic contractions have been superimposed, the oscilloscope trace being retriggered immediately after cessation of the previous sweep. The increasing failure of relaxation is seen as increasing inability of the trace to return to base-line at the end of voluntary contraction, so that the next contraction starts from an increasingly less relaxed position. The duration of maximal voluntary contraction is $6 \mathrm{~s}$, as indicated by the bar.

retain some stiffness. Absence of EMG activity was confirmed in one experiment on ADM using a concentric needle electrode. That membrane irritability as reflected in myotonic EMG activity does not fully explain the mechanical failure of relaxation was investigated in FDP in four experimental sessions, using both surface and needle electrodes.

With the fingers prevented from flexing, vigorous (isometric) contraction of FDP was followed by typical myotonic EMG activity. Once the EMG activity (as recorded by the concentric needle) had subsided, the restraint was removed and immediate finger flexion resulted. Re-extension of the fingers under the influence of the soft steel spring occurred slowly after some delay, even though the spring had been capable of extending the fingers prior to contraction. Alternatively, restraint was removed on cessation of voluntary contraction, with resultant finger flexion which was maintained long after the EMG activity had subsided. The findings were essentially similar with isotonic contractions of FDP, finger flexion being maintained by the myotonic contraction on cessation

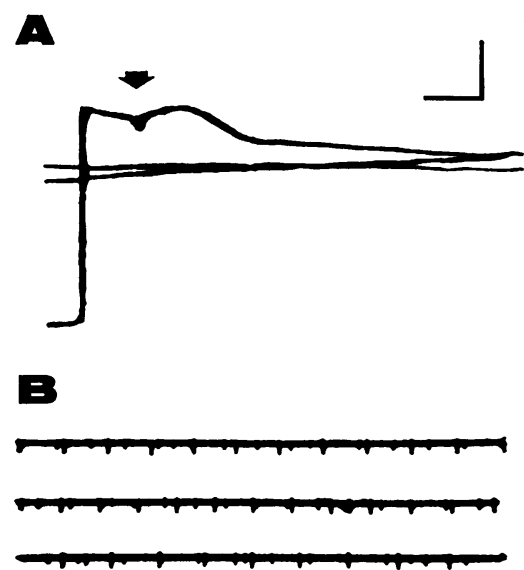

FIG. 5. Isotonic contraction of FDP. A: displacement record. Initially the fingers are maintained in an extended position by the mild steel spring but are then flexed in a maximal voluntary contraction of FDP which is maintained for $5 \mathrm{~s}$, ending at the arrow. On cessation of voluntary contraction the fingers remain flexed, extending only partially and then very slowly under the influence of the spring. The oscilloscope trace was retriggered twice to show the persistent failure of relaxation. B: EMG recorded with a concentric needle electrode during the second of the above oscilloscope sweeps showing only very low voltage EMG activity (less than $40 \mu \mathrm{V}$, mostly of positive sharp wave form). Failure of relaxation outlasts significant EMG activity. Calibrations: horizontal-5 $s$ for A, $1 s$ for B; vertical $-150 \mu \mathrm{V}$ for B.

of voluntary contraction, and re-extension occurring slowly under the influence of the spring long after the subsidence of significant EMG activity (as recorded by the needle electrode). Figure 5 illustrates this phenomenon. Isotonic voluntary contraction starts from a relaxed position and ceases after $5 \mathrm{~s}$ at the arrow. Myotonic EMG activity decreased over the succeeding $12 \mathrm{~s}$, and thereafter only an occasional potential of very low voltage was recorded (Fig. 5B). The fingers remain partially flexed for more than $100 \mathrm{~s}$, flexion being maintained against a spring which originally had been capable of holding the fingers at the starting position. During this phase of 'non-electrical' muscle stiffness the fingers could be passively re-extended by the examiner. Following partial extension the fingers were capable of maintaining the new position to which they had been moved for some time, per- 
haps flexing up slightly or extending slightly with the passage of time. If the fingers were extended to the control position and immediately released they commonly flexed again without significant audible or visible EMG activity.

The distinction between the electrical and mechanical components of myotonia could be appreciated in the fatigued state. Clinical myotonia decreased during muscle fatigue, at times even disappearing, but the EMG evidence of myotonia appeared to be more affected than the mechanical delay in relaxation. On a number of occasions the fatigued muscle demonstrated what appeared clinically to be myotonia but this was not accompanied by EMG activity. Studies with the needle electrode indicated that as the muscle fatigued the EMG signs of hyperexcitability became less prominent and ultimately disappeared.

EFFECT OF COOLING Muscle cooling did not provoke spontaneous EMG activity and did not produce a spontaneous muscle contraction. In four experiments in the two subjects cooling resulted in a profound prolongation of the isometric twitch times of $\mathrm{ADM}$, the changes being more prominent for the half-relaxation time than for the contraction time (Fig. 6; compare with Fig. 4, Burke et al., 1974b). Surface temperature decreased from $34-35^{\circ}$ to between $21^{\circ} \mathrm{C}$ and $28^{\circ} \mathrm{C}$ in different experiments. The rate of increase of half-relaxation time, $20-28 \%$ (mean $23.5 \%$ ) for each ${ }^{\circ} \mathrm{C}$ fall in temperature, greatly exceeded that of the contraction time, $11 \%-12.5 \%$ (mean $11.5 \%$ ) per ${ }^{\circ} \mathrm{C}$ fall in tem-

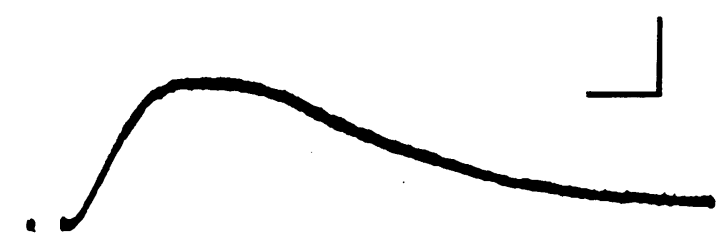

FIG. 6. Effect of cooling on isometric twitch times of ADM. Surface temperature measured over ADM was decreased to $21^{\circ} \mathrm{C}$, resulting in prolongation of the contraction time to $150 \mathrm{~ms}$ and of the half-relaxation time to $270 \mathrm{~ms}$ (compare with Fig. 5, Burke et al., 1974b). Calibrations: horizontal-100 ms; vertical$46.6 \times 10^{-5} \mathrm{Nm}$. perature, so that the ratio of half-relaxation time to contraction time increased to 1.9 at $21^{\circ} \mathrm{C}$. Since changes in skin temperature probably exceed changes in muscle temperature, these figures probably underestimate the effects of cooling. The evoked muscle action potential remained a discrete potential. There was no evidence of repetitive EMG activity with surface electrodes at gains of $150 \mu \mathrm{V} / \mathrm{cm}$, so that the marked prolongation of half-relaxation time cannot be attributed to electrical myotonia.

During cooling, repetitive electrical activation, even tetanization, of ADM failed to provoke mechanical or EMG evidence of myotonia. With voluntary contraction, muscle cooling appeared initially to aggravate both the electrical and the mechanical evidence of myotonia but, since it also aggravated the fatigue process, this effect could not be assessed adequately. However, as the fatiguing process became dominant the EMG evidence of hyperexcitability disappeared, so that it was again possible to record a greatly delayed relaxation of the cooled fatigued ADM in the absence of electrical activity.

\section{DISCUSSION}

In the two subjects with paramyotonia congenita, the adequate stimulus for myotonia appears to be vigorous voluntary contraction, preferably repeated voluntary contraction, and isotonic rather than isometric voluntary contraction. That myotonia could not be demonstrated after electrically induced contraction was unexpected and cannot be adequately explained. Possibly the synchronized contraction of motor units in a tetanus is a less effective stimulus than the asynchronous discharge of motor units produced by voluntary contraction.

Although systematic observations were not made, voluntary contraction of ADM produced in both control and experimental subjects a greater torque than tetanic contraction, a finding at variance with that of Merton (1954) who studied adductor pollicis. The torque produced by voluntary abduction of digit 5 depends not only on ADM but also on the flexor carpi ulnaris which stabilizes the pisiform bone, thus contributing indirectly to the recorded torque. Differences in the strength of voluntary and 
tetanic contraction therefore do not explain the greater efficacy of voluntary contraction in producing myotonia in ADM. Certainly myotonia became more difficult to elicit as the muscle fatigued: perhaps this explains why some authors have reported that myotonia is not paradoxical in some patients with paramyotonia. In any event, the force produced by muscle contraction cannot be the major determinant of myotonia because contractile force increases as muscle length increases (Joyce et al., 1969), so that isometric contraction should have produced myotonia more readily than isotonic contraction. During maximal voluntary contraction motor units commonly discharge at frequencies of 60 $80 / \mathrm{s}$, and some exceed 100/s (Marsden et al., 1971), although such rates cannot be sustained (Tanji and Kato, 1972; Hannerz, 1973). Presumably, synchronized firing of motor units at $50 / \mathrm{s}$ as in a tetanus is a relatively ineffective stimulus for myotonia.

In both experimental subjects the delayed muscular relaxation which produces clinical myotonia appeared to have two components. The EMG signs of myotonia are similar to those found in other myotonic disorders. A membrane phenomenon has been implicated as the cause of this 'electrical myotonia' and, indeed, experimental myotonia can be produced by agents which interfere with muscle cell membrane stability-for example, 20, 25-diazacholesterol (Bryant, 1973). In paramyotonia muscle fatigue appears to arise from a progressive decrease in excitability of the muscle cell membrane (Burke et al., 1974b), and, not surprisingly therefore, the EMG signs of myotonia decrease as fatigue progresses. The loss of 'electrical myotonia' with fatigue of muscle explains the EMG silence found in paramyotonia after cooling because cooling greatly aggravates the fatiguing process (Burke et al., 1974b). That the inability to relax after a vigorous contraction outlasts the classical EMG signs of myotonia suggests that, while there may be a membrane defect, an additional mechanical factor contributes to clinical myotonia. A primary defect of the ability to relax of the contractile mechanism within the muscle cell is therefore suggested. A similar conclusion was reached by Haynes and Thrush (1972) based on in vitro studies of membrane phenomena: '... the myotonia itself is possibly a direct effect of the disease upon the contractile elements of the muscle'.

If clinical myotonia has two components, electrical and mechanical, due to two defects, membrane and contractile, an hypothesis can be advanced to explain why isotonic contractions appear to produce clinical myotonia more readily than isometric contractions. With isometric contraction realignment of contractile filaments would be limited, but when significant shortening occurs, as in an isotonic contraction, complete realignment of contractile filaments must take place. As a result, the mechanical component of myotonia, that due to a defect in the ability of contractile elements to 'relax', would be more prominent in isotonic contractions.

The effect of muscle cooling on the twitch times of ADM provides additional support for the presence of a mechanical factor in myotonia. There are few observations on the effect of cooling on twitch characteristics of human muscle. In excised human muscle at $20^{\circ} \mathrm{C}$ Brust and Cosla (1967) reported contraction times of $188.3 \mathrm{~ms}$ and $381.2 \mathrm{~ms}$ and half relaxation times of $252.2 \mathrm{~ms}$ and $597 \cdot 4 \mathrm{~ms}$ for groups of muscles classified respectively as fast and slow. Buchthal and Schmalbruch (1970) found in intact human muscle that contraction times of fast fibres increased by up to $10 \%$ per ${ }^{\circ} \mathrm{C}$ and of slow fibres by up to $7 \%$ per ${ }^{\circ} \mathrm{C}$. It is difficult to compare these data with those of the present study, since the latter were obtained using surface temperature measurements and therefore probably underestimate the rate of increase in twitch times. Nevertheless, the rate of increase in contraction time was of a similar order of magnitude to that reported by Buchthal and Schmalbruch (1970), who unfortunately did not report values for the half-relaxation time. From the data of Brust and Cosla (1967) the ratio of half-relaxation time to contraction time at $20^{\circ} \mathrm{C}$ is 1.34 for fast muscles and 1.57 for slow muscles, figures which are significantly less than the ratio of 1.9 found in the present study, even though the effective temperature was probably significantly higher in the present study. These data suggest that, although the changes in contraction time induced by cooling may be acceptable as within normal limits, the increase in half-relaxation time is probably excessive. The findings are 
therefore consistent with a defect of the contractile mechanism within the muscle cell, a defect demonstrable at room temperature only after vigorous voluntary contraction, but more readily demonstrated in cooler circumstances. As with the fatiguing process (Burke et al., 1974b), cooling appears to lower the threshold for the demonstration of the muscular defect-it does not appear to play a primary pathogenic role.

The suggestion that the myotonia of paramyotonia congenita has two components, a membrane ('electrical') component and an internal contractile ('mechanical') component, raises therapeutic implications. It is not surprising that drugs which stabilize cell membrane function often prove disappointing clinically in the treatment of paramyotonia, since such agents would prove beneficial only if membrane phenomena contribute significantly to the failure of relaxation. Indeed, it is quite conceivable that, as far as the myotonia is concerned, the electrical phenomena are of little clinical relevance apart from providing an important diagnostic sign for the electromyographer.

\section{SUMMARY}

Myotonia was studied in two brothers with paramyotonia congenita. In non-fatigued muscle, myotonia could be elicited at room temperature by vigorous voluntary contraction, more readily under isotonic than isometric conditions. With repeated voluntary contractions the myotonia increased in successive contractions ('paradoxical myotonia') until the development of muscle fatigue resulted in its disappearance. Electrical stimulation, including tetanization at $50 \mathrm{~Hz}$, failed to elicit myotonia. It was frequently noted that the EMG evidence of myotonia subsided before the contracting muscle had relaxed completely.

Muscle cooling appeared initially to aggravate the myotonia but, since it also aggravated the fatigue process, evidence of myotonia subsequently disappeared. Isometric twitch times of the abductor digiti minimi were prolonged by muscle cooling, the half-relaxation time being affected more than the contraction time. No repetitive EMG activity accompanied these changes.
These results are best explained by postulating that in paramyotonia congenita the myotonic failure of relaxation has two componentsexcessive irritability of the muscle cell membrane which produces the classical EMG features of myotonia, and a defect of the ability of the contractile mechanism within the muscle cell to relax after contraction. This conclusion explains the limited therapeutic efficacy of drugs which act by stabilizing membranes.

The authors are grateful to Mr Peter van Megen and A.C.I., Ltd, without whose generous assistance these studies could not have been performed, and to Professor J. W. Lance for advice in the preparation of this report. Illustrations were photographed by the Department of Medical Illustration, University of N.S.W.

\section{REFERENCES}

Brust, M., and Cosla, H. W. (1967). Contractility of isolated human skeletal muscle. Archives of Physical Medicine and Rehabilitation, 48, 543-555.

Bryant, S. H. (1973). The electrophysiology of myotonia, with a review of congenital myotonia of goats. In $\mathrm{New}$ Developments in Electromyography and Clinical Neurophysiology, vol. 1, pp. 420-450. Edited by J. E. Desmedt. Karger: Basel.

Buchthal, F., and Schmalbruch, H. (1970). Contraction times and fibre types in intact human muscle. Acta Physiologica Scandinavica, 79, 435-452.

Burke, D., Skuse, N. F., and Lethlean, A. K. (1974a), Isometric contraction of the abductor digiti minimi muscle in man. Journal of Neurology, Neurosurgery, and Psychiatry. (In press.)

Burke, D., Skuse, N. F., and Lethlean, A. K. (1974b). The contractile properties of the abductor digiti minimi muscle in paramyotonia congenita. Journal of Neurology, Neurosurgery, and Psychiatry, 37, 894-899.

Hannerz, J. (1973). Discharge properties of motor units in man. Experientia, 29, 45-46.

Haynes, J., and Thrush, D. C. (1972). Paramyotonia congenita: an electrophysiological study. Brain, 95, 553-558.

Joyce, G C., Rack, P. M. H., and Westbury, D. R. (1969). The mechanical properties of cat soleus muscle during controlled lengthening and shortening movements. Journal of Physiology, 204, 461-474.

Marsden, C. D., Meadows, J. C., and Merton, P. A. (1971). Isolated single motor units in human muscle and their rate of discharge during maximal voluntary effort. Journal of Physiology, 217, 12-13P.

Merton, P. A. (1954). Voluntary strength and fatigue. Journal of Physiology, 123, 553-564.

Preswick, G, Stewart, R. D. H., O'Hara, P., and Murray, I. P. C. (1966). The value of muscle twitch and Achilles reflex recordings in thyroid disorders. Medical Journal of Australia, 1, 473-477.

Tanji, J., and Kato, M. (1972). Discharges of single motor units at voluntary contraction of abductor digiti minimi muscle in man. Brain Research, 45, 590-593. 